\title{
ON CLANS OF NON-NEGATIVE MATRICES
}

\author{
DENNISON R. BROWN ${ }^{1}$
}

A clan is a compact connected topological semigroup with identity. Professor A. D. Wallace has raised the following question [9]: Is a clan of real $n \times n$ matrices with non-negative entries, which contains the identity matrix, necessarily acyclic? That is to say, do all of the Alexander-Cech cohomology groups with arbitrary coefficients (in positive dimensions) vanish? In this paper the slightly stronger result, that any non-negative matrix clan is contractible, is obtained. This follows from the result, interesting in itself, that a compact group of non-negative matrices is finite (Theorem 2).

The author wishes here to express his sincere gratitude to Professor R. J. Koch for his helpful advice and encouragement.

The set of order $n$ non-negative matrices is denoted by $N_{n}$. The real and complex general linear groups of order $n$ are represented by $\mathrm{Gl}(n, R)$ and $\mathrm{Gl}(n, C)$, respectively. The semigroup terminology used is that of [8]; in particular, $K$ denotes the minimal ideal of a clan $S, E$ denotes the set of idempotents of $S$, and for $e \in E, H(e)$ is the maximal subgroup of $S$ containing $e$. An iseomorphism is an isomorphism which is also a homeomorphism. The topology of $N_{n}$ is any locally convex topology; for example, the topology of Euclidean $n^{2}$ space.

The equation $M=\operatorname{diag}(A, B)$ means that $M$ is the matrix which, in $2 \times 2$ block form, has the square submatrix $A$ in the upper left corner, the square submatrix $B$ in the lower right corner, and zero entries elsewhere. The $k \times k$ identity matrix is denoted by $I_{k}$ when used as a submatrix. The set of eigenvalues of a matrix $M$ is denoted by $S(M)$.

The well-known theorem $[1$, p. 80$]$ that a non-negative matrix $M$ has a real eigenvalue $r$ such that if $\lambda \in S(M)$, then $|\lambda| \leqq r$ is used without proof. Also used without proof is the following theorem, due to Karpelevich [3], and stated in less than full generality:

TheOREM 1. Let $M \in N_{n}$, and let $M$ have maximal real eigenvalue 1. If $\lambda \in S(M),|\lambda|=1$, then $\lambda^{k}=1$ for some $k \leqq n$.

Presented to the Society, November 16, 1962; received by the editors May 25, 1963.

${ }^{1}$ This work was supported in part under NSF Contract G-14085, and is part of a dissertation presented to the Graduate Faculty of Louisiana State University. 
Lemma 1. Let $X \in G$, a compact subgroup of $\mathrm{Gl}(n, C)$. If $\lambda \in S(X)$, then $|\lambda|=1$.

Proof. The determinant function maps $G$ homomorphically into the unit circle. Hence $1=|\operatorname{det} X|=\left|\lambda_{1} \lambda_{2} \cdots \lambda_{n}\right|, \lambda_{i} \in S(X)$. Let $P \in \operatorname{Gl}(n, C)$ such that $A=P X P^{-1}$ is triangular, diagonal $A=\left(\lambda_{1}, \lambda_{2}, \cdots, \lambda_{n}\right)$. Since diagonal $A^{t}=\left(\lambda_{1}^{t}, \lambda_{2}^{t}, \cdots, \lambda_{n}^{t}\right)$ and the group $P G P^{-1}$ is compact, if follows that $\left|\lambda_{i}\right| \leqq 1, i=1, \cdots, n$. This is clearly sufficient.

THEOREM 2. Let $H(e)$ be a compact topological group, $H(e) \subset N_{n}$. Then $H(e)$ is finite.

Proof. Define $f: H(e) \rightarrow \operatorname{Gl}(n, R)$ by $f(x)=x+I-e$. The function $f$ is clearly an iseomorphism. Since $f(H(e))$ is a compact subgroup of $\mathrm{Gl}(n, R), H(e)$ is a Lie group. The identity component $C$ of $H(e)$ is therefore open; hence it suffices to prove that $H(e)$ is totally disconnected. If $C \neq\{e\}$, then $C$ has a nontrivial one parameter group [5, p. 105], hence elements of infinite order. The proof is then completed by contradiction when it is shown that every element of $H(e)$ has finite order.

Let $X \in H(e)$. There exists $B \in G l(n, R)$ such that $B e B^{-1}$ $=\operatorname{diag}\left(I_{k}, 0\right)$, where rank $e$ is assumed equal to $k$. Since $B e B^{-1}$ is an identity for $B X B^{-1}, B X B^{-1}=\operatorname{diag}\left(X_{k}, 0\right)$, where $X_{k}$ is a rank $k$ real $k \times k$ matrix. Let $f$ be the iseomorphism of $B H(e) B^{-1}$ into $\operatorname{Gl}(n, R)$ defined by $f\left(B X B^{-1}\right)=B X B^{-1}+I-B e B^{-1}$. Since $f\left(B H(e) B^{-1}\right)$ is iseomorphic to $H(e)$, it suffices to find an integer $m$ such that $f\left(B X B^{-1}\right)^{m}=f\left(B e B^{-1}\right)=I$.

Assume $k<n$. Note $S(X)=S\left(B X B^{-1}\right)=S\left(f\left(B X B^{-1}\right)\right) \cup\{0\}$. For if $\lambda \in S\left(B X B^{-1}\right), \lambda \neq 0$, then $\operatorname{det}\left(X_{k}-\lambda I_{k}\right)=0$. Hence

$$
\operatorname{det}\left(f\left(B X B^{-1}\right)-\lambda I\right)=(1-\lambda)^{n-k} \cdot \operatorname{det}\left(X_{k}-\lambda I_{k}\right)=0
$$

and

$$
\lambda \in S\left(f\left(B X B^{-1}\right)\right) .
$$

Conversely, if $\lambda \neq 1$ and $\lambda \in S\left(f\left(B X B^{-1}\right)\right)$, then $\lambda \in S\left(B X B^{-1}\right)$. Finally, by Lemma $1, \lambda \in S\left(f\left(B X B^{-1}\right)\right)$ gives $|\lambda|=1$; therefore $\lambda \in S\left(B X B^{-1}\right)$, $\lambda \neq 0$ also yields $|\lambda|=1$. Since $X \in N_{n}, 1 \in S\left(B X B^{-1}\right)$, and $S\left(B X B^{-1}\right)$ $=S\left(f\left(B X B^{-1}\right)\right) \cup\{0\}$. By Theorem $1, S\left(B X B^{-1}\right) \subset\left\{\lambda: \lambda^{t}=1, t \leqq n\right\}$ $\cup\{0\}$. If $k=n$, a similar argument can be given. In either event $S\left(f\left(B X B^{-1}\right)\right) \subset\left\{\lambda: \lambda^{t}=1, t \leqq n\right\}$. Let $P \in \operatorname{Gl}(n, C)$ such that $D=P f\left(B X B^{-1}\right) P^{-1}$ is lower triangular and diagonal $D=\left\{\lambda_{1}, \lambda_{2}, \cdots\right.$, $\left.\lambda_{n}\right\}$. Note $\lambda_{i} \in S\left(f\left(B X B^{-1}\right)\right), i=1, \cdots, n$. Let $m=$ least common multiple $\left\{t_{i}: \lambda_{i}^{t_{i}}=1, t_{i} \leqq n\right\}$. Then diagonal $D^{m}=\{1,1, \cdots, 1\}$. Now 
if $j=i-1$, then $\left(D^{m p}\right)_{i j}=p \cdot\left(D^{m}\right)_{i j}$. Hence, by the compactness of $P f\left(B H(e) B^{-1}\right) P^{-1},\left(D^{m}\right)_{i j}=0, j=i-1$. By a straightforward induction, it follows that $\left(D^{m}\right)_{i j}=0, j<i, i=1, \cdots, n$. Hence $D^{m}=I$, and therefore $f\left(B X B^{-1}\right)$ has order $\leqq m$, which completes the proof.

Corollary 1. Let $S$ be a continuum semigroup in $N_{n}$. Then $K \subset E$.

Proof. Fix $e \in E \cap K$. Then $e S e=H(e)$ [8]. Since $e S e$ is a continuum, it is degenerate; hence $H(e)=\{e\}$. The corollary now follows from the fact that $K=\bigcup\{H(e): e \in K\}$.

If $S$ is a clan, it is known [8] that $H^{n}(S)=H^{n}(e S e)$ for $e \in K \cap E$, $n \geqq 0$. If, also, $S \subset N_{n}$, then by Theorem $2, H^{n}(S)=H^{n}(\{e\})=0, n>0$. Hence $S$ is acyclic. It will now be shown that $S$ is contractible. The following lemma is due to Gluskin [2].

Leмma 2. Let $S$ be an $n \times n$ complex matrix semigroup. Let $e, f \in E$ and $f \in e S e$. If $f \neq e$, then $\operatorname{rank} f<\operatorname{rank} e$.

Proof. Suppose rank $e=r, e \neq f$. Choose $v$ such that $v e v^{-1}$ $=\operatorname{diag}\left(I_{r}, 0\right)$. Then $v f v^{-1}=\operatorname{diag}(g, 0)$, since $e$ is an identity for $f$. Note $g$ is an $r \times r$ complex matrix, and $g^{2}=g$. Since $\operatorname{rank} v f v^{-1}=\operatorname{rank} f$, it suffices to show $\operatorname{det}(g)=0$. If this is not the case, then $g$ is an idempotent in $\operatorname{Gl}(r, C)$; hence $g=I_{r}$. But this implies $f=e$, contrary to assumption. This completes the proof.

An I-semigroup is a clan on an interval such that one endpoint is an identity and the other a zero. It is shown in [6] that the only types of $I$-semigroups are the following: (i) $S$ has the multiplication of the real interval $[0,1]$; (ii) $S$ has a multiplication isomorphic to the interval $[1 / 2,1]$ under the operation $x \circ y=\max \{1 / 2, x y\}$; (iii) $S$ is idempotent and has a multiplication isomorphic to the interval $[0,1]$ under the operation $x \circ y=\min \{x, y\}$; (iv) $S$ is the union of a collection of semigroups of types (i), (ii), and (iii) which meet only at their respective endpoints.

LEMMA 3. Let $S$ be a clan in which, for each $e \in E, H(e)$ is totally disconnected. Suppose also that there exists a neighborhood $V$ of 1 such that $V \cap E=1$. Then there is an I-semigroup in $S$ having 1 as an identity.

Proof. It is well known [7] that the existence of the neighborhood $V$ above is sufficient to insure a local one-parameter semigroup $\sigma([0,1])$ in $V$ such that $\sigma(0)=1, \sigma(a) \notin H(1), 0<a \leqq 1$, and if $\sigma(a)$ $=\sigma(b) g, g \in N(1)$, then $a=b$ and $g=1$. In the same paper, it is shown that $\sigma$ can be extended to a full one-parameter semigroup by defining $\sigma(t)=\sigma(1) \sigma(t-1)$ for $t \in[1,2]$ and proceeding inductively. Now the closure of $\sigma([0, \infty))$ is a commutative clan, hence its minimal ideal is 
a connected group, and therefore a single point. It follows by a theorem of Koch [4] that this clan has exactly 2 idempotents and is an $I$-semigroup.

THEOREM 3. Let $S$ be a nondegenerate clan in $N_{n}$. Then $S$ contains an $I$-semigroup from 1 to $K$, and $S$ is contractible.

Proof. By Lemma 2, there exists a neighborhood $V$ of 1 containing no other idempotents; this follows from the fact that the rank of an idempotent equals its trace. By Theorem 2 each $H(e)$ is finite. It follows from Lemma 3 that there exists an $I$-semigroup from 1 to $e \in E$. By Lemma 2, rank $e<\operatorname{rank} 1$. If $e \notin K$, then $e S e$ is a nondegenerate subclan with identity $e$, and the above argument produces an $I$-semigroup from $e$ to $f \in E$, rank $f<\operatorname{rank} e$. In this manner, an idempotent of minimal rank in $S$ is obtained, which clearly belongs to $K$. The union of the $I$-semigroups constructed above is the desired $I$-semigroup.

Let $T$ be an $I$-semigroup in $S$ with endpoints 1 and $e \in K \cap E$. Define $F: S \times T \rightarrow S$ by $F(x, t)=t x t$. Then $F(x, 1)=x$, and $F(x, e)$ $=e x e=e$, for each $x \in S$. Hence $S$ is contractible. This completes the proof.

By Lemma 2, no $I$-semigroup in $N_{n}$ can be of type (iii) mentioned above. On the other hand, it is well known that if $A$ is a nilpotent $n \times n$ complex matrix, then $A^{n}=0$. It follows that the $I$-semigroups in $N_{n}$ are either of type (i), or of type (iv), constructed by joining together the endpoints of semigroups of type (i).

\section{BIBLIOGRAPHY}

1. F. R. Gantmacher, Applications of the theory of matrices, Interscience, New York, 1959; pp. 61-105.

2. L. M. Gluskin, On matrix semigroups, Izv. Akad. Nauk 22 (1958), 439-448.

3. F. I. Karpelevich, On the characteristic roots of matrices with non-negative elements, Izv. Akad. Nauk 15 (1951), 361-383.

4. R. J. Koch, On topological semigroups, Dissertation, Tulane University, New Orleans, La., 1953.

5. D. Montgomery and L. Zippin, Topological transformation groups, Interscience, New York, 1955; p. 105.

6. P. S. Mostert and A. L. Shields, On the structure of semigroups on a compact manifold with boundary, Ann. of Math. 65 (1957), 117-143.

7. - One parameter semigroups in a semigroup, Trans. Amer. Math. Soc. 96 (1960), 510-517.

8. A. D. Wallace, The structure of topological semigroups, Bull. Amer. Math. Soc. 56 (1955), 95-112.

9. - Problems concerning semigroups, Bull. Amer. Math. Soc. 68 (1962), $447-448$.

Louisiana State University 